\title{
Pourquoi un dossier dédié aux cancers digestifs rares ?
}

\section{Why an Edition Devoted to Rare Cancers of the Digestive System?}

\author{
S. Manfredi (Secrétaire adjoint de la Fédération Francophone de Cancérologie Digestive - FFCD) \\ (C) Lavoisier SAS 2018
}

Après quatre numéros spéciaux d'Oncologie consacrés aux cancers digestifs les plus fréquents (cancer du côlon en 2014, cancers œsogastriques en 2013, cancer du pancréas en 2015 et cancer hépatocellulaire en 2017), il nous a paru nécessaire de consacrer un numéro aux cancers digestifs plus rares.

Parmi les cancers digestifs rares, nous en avons sélectionné trois qui par leur présentation, leur prise en charge et leur pronostic présentent des originalités importantes à connaître.

Les trois cancers traités dans ce numéro bien que rares en termes d'incidence font partie du quotidien des gastroentérologues, des gastroentérologues spécialisés en oncologie et des oncologues médicaux ou radiothérapeutes.

Ces trois localisations cancéreuses illustrent parfaitement la nécessaire multidisciplinarité et la concertation des différents professionnels requises pour la prise en charge de tout cancer.

Le cancer du canal anal fait régulièrement intervenir l'expertise du gastroentérologue, du proctologue, de l'oncologue, du radiothérapeute, du chirurgien, mais aussi de l'infectiologue dans nombre de cas. Ce cancer digestif rare peut être considéré comme un modèle de cancer radiochimiosensible permettant dans une majorité de cas d'éviter une chirurgie mutilante. La meilleure connaissance récente de la carcinogenèse du cancer du canal anal permet de développer des stratégies de prévention par vaccination.

Les lymphomes digestifs requièrent l'expertise des gastroentérologues, des oncologues et en particulier des hématologues, moins souvent des chirurgiens et des radiothérapeutes.
Ces cancers sont considérés comme chimiosensibles. Certains, liés à l'infection par Helicobacter pylori, peuvent même être traités et guéris par antibiothérapie. La coordination de RCP (réunion de concertation pluridisciplinaire) de cancérologie digestive et de RCP d'hématologie est primordiale.

Les tumeurs stromales gastro-intestinales (GIST) sont également originales et nécessitent l'expertise du gastroentérologue, du chirurgien, de l'anatomopathologiste, du biologiste moléculaire, de l'oncologue en particulier spécialisé en sarcome. Là aussi, la coordination de RCP de cancérologie digestive et de RCP sarcomes participant au réseau NetSARC est nécessaire. Les GIST ont été les premiers cancers à répondre à une thérapie ciblée et peuvent à ce compte être considérées comme un modèle.

Ces trois localisations cancéreuses présentent des originalités qu'il est important de connaître afin de traiter au mieux nos patients. L'objectif de ce numéro spécial est de réaliser une mise au point synthétique de la prise en charge de ces trois cancers au pronostic plutôt favorable.

Nous avons demandé à de jeunes médecins (internes, chefs de cliniques et jeunes praticiens hospitaliers) de rédiger ce numéro sous la supervision de seniors.

J'espère que ces mises au point vous combleront, vous apporteront les bases de la prise en charge de ces cancers et finiront par vous convaincre de la nécessité de collaborations actives multidisciplinaires.

Bonne lecture !

S. Manfredi $(\square)$

Service d'hépatogastroentérologie et oncologie digestive,

CHU Le Bocage, F-21000 Dijon, France

e-mail : sylvain.manfredi@chu-dijon.fr 